Exp. Anim. 62(3), 229-235, 2013

\title{
-Original-
}

\section{Effect of Post-Weaning Individual Housing on Autonomic Responses in Male Rats to Sexually Receptive Female Rats}

\author{
Hideaki INAGAKI, Masayoshi KUWAHARA, and Hirokazu TSUBONE \\ Department of Comparative Pathophysiology, Graduate School of Agricultural and Life Sciences, The University \\ of Tokyo, 1-1-1 Yayoi, Bunkyo-ku, Tokyo 113-8657, Japan
}

\begin{abstract}
Post-weaning individual housing induces significant alterations in the reward system of adult male rats presented with sexually receptive female rats. In this study, we examined the effects of post-weaning individual housing on autonomic nervous activity in adult male rats during encounters with sexually receptive female rats to assess whether different affective states depending on postweaning housing conditions are produced. Changes in heart rate and spectral parameters of heart rate variability indicated that in post-weaning individually housed male rats, both sympathetic and parasympathetic activity increased with no change in the sympathovagal balance, while in postweaning socially housed male rats, both sympathetic and parasympathetic activity decreased with a predominance of parasympathetic activity. These two patterns of shifts in sympathovagal balances closely resembled changes in autonomic nervous activity with regard to classical appetitive conditioning in male rats. The autonomic changes in male rats housed individually after weaning corresponded to changes associated with the reward-expecting state evoked by the conditioned stimulus, and the autonomic changes observed in male rats housed socially after weaning corresponded to changes associated with the reward-receiving state evoked by the unconditioned stimulus. These results suggest that different affective states were induced in adult male rats during sexual encounters depending on male-male social interactions after weaning. The remarkable change caused by postweaning individual housing may be ascribed to alteration of the reward system during sexual encounters induced by deficiency of intermale social communication after weaning.
\end{abstract}

Key words: early social isolation, positive affective state, power spectral analysis of heart rate variability, social communication

\section{Introduction}

Post-weaning individual housing induces significant alterations in social behaviors of laboratory rats compared with socially housed (i.e., group-housed) rats [1, $3,5-7,15,20,21]$. Our previous study showed that adult male rats that were housed individually after weaning emitted fewer ultrasonic vocalizations referred to as " 50 $\mathrm{kHz}$ calls" compared with rats housed socially after weaning that encountered sexually receptive female rats [11]. Adult rats use ultrasonic vocalizations as the primary method of communicating social affective states, and $50-\mathrm{kHz}$ calls are considered to be positively correlated with positive and social affective states $[4,12,16]$. Thus, our previous finding that post-weaning individual housing attenuated female-induced male ultrasonic vocalizations suggested an altered function of the reward system in male rats during a sexual encounter. Based on

(Received 21 January 2013 / Accepted 6 March 2013)

Address corresponding: H. Inagaki, Department of Veterinary Ethology, Graduate School of Agricultural and Life Sciences, The University of

Tokyo, 1-1-1 Yayoi, Bunkyo-ku, Tokyo 113-8657, Japan

(C)2013 Japanese Association for Laboratory Animal Science 
these results, we hypothesized that different affective states according to post-weaning housing conditions are produced in adult male rats by sexually receptive female rats.

To test this hypothesis, we examined changes in autonomic nervous control of the heart as indices of differences in affective states induced by sexually receptive female rats. It was previously shown in humans and laboratory rats that characteristic changes in the cardiac sympathovagal balance may be associated with distinct affective states induced by presentation of stress or reward stimuli $[9,10,13]$, indicating that changes in autonomic control of the heart can be used as reliable indices to distinguish affective states.

\section{Materials and Methods}

\section{Subjects}

Sixteen male Wistar rats (Saitama Experimental Animals Supply, Saitama, Japan) were used. The animals were provided with water and food ad libitum and kept on a 12-h light-dark cycle with lights off at 20:00. The vivarium was maintained at a constant temperature (24 $\pm 1{ }^{\circ} \mathrm{C}$ ) and humidity $(40-45 \%)$. All subjects were weaned at 21 days of age. After weaning, each was assigned to one of two groups. In the individually housed group, eight subjects were kept individually for 6 months after weaning in wire-topped, transparent cages $(300 \times$ $200 \times 150 \mathrm{~mm}$ ) with wood shavings for bedding. These animals were deprived of physical contact, while still having olfactory, auditory, and visual contact. The eight rats in the other group were housed socially (four pairs) for the same period in wire-topped, transparent cages $(400 \times 250 \times 180 \mathrm{~mm})$ with wood shavings for bedding.

\section{Experimental apparatus and procedures}

First, a telemetry electrocardiogram (ECG) transmitter (TA10EA-F20; Data Sciences International, St. Paul, MN, USA) was implanted in each rat. Surgery was performed under intraperitoneal pentobarbital sodium anesthesia $(50 \mathrm{mg} / \mathrm{kg})$. The transmitter body was placed in the abdominal cavity, and the paired wire electrodes were fixed under the skin of the ventral thorax near the right atrium and apex cordis, respectively. After surgery, each rat was kept individually in a wire-topped transparent cage $(400 \times 250 \times 180 \mathrm{~mm})$ with wood shavings for bedding, and each cage was placed in an incubator (MIR553; Sanyo, Tokyo, Japan) maintained at a constant temperature $\left(24 \pm 1^{\circ} \mathrm{C}\right)$ and humidity $(40-45 \%)$. Animals were provided with water and food ad libitum under a 12-h light-dark cycle with lights off at 20:00. Ten days after surgery, the rats were used in the experiment.

Experiments were conducted during the first half of the animals' dark cycle under dim red illumination (20 W). All experimental procedures were carried out in the incubator in which the home cage of each subject was placed. The door of the incubator was soundproofed and had a transparent window $(800 \times 200 \mathrm{~mm})$. For the experiment, a video camera (DCR-VX1000; Sony, Tokyo, Japan) was placed in front of the incubator window. In addition, a signal-receiving board (RA1610; Data Science Automation, Pittsburgh, PA, USA) was set under the cage. Before the test session, ECG signals were recorded for 5 min using an ECG processor (SBP2000; Softron, Tokyo, Japan) to create baseline data. Subsequently, an empty wire mesh box (WMB; $80 \times 80 \times 200$ $\mathrm{mm}$, size of opening $8 \times 8 \mathrm{~mm}$ ) was placed inside the home cage of each subject, and ECG signals were recorded for $15 \mathrm{~min}$ and stored in a computer. Rat behavior was simultaneously recorded using the video camera. After an interval of $30 \mathrm{~min}$, we put an ovariectomized female rat (OVX) ( 3 months of age, at least 10 days after surgery, from groups of three per the same type of transparent cage used for housing socially housed male rats) into another wire mesh box (the same as described above) and placed the wire mesh box containing the OVX rat inside the home cage of each subject. Immediately afterward, we began recording the ECG and behavior data and continued this for $15 \mathrm{~min}$. After an interval of $30 \mathrm{~min}$, we put a proestrous female rat (PES) (under the same housing conditions as those of OVX rats) into another wire mesh box and placed the wire mesh box containing the PES rat inside the home cage of each subject to record data (ECG signals and behaviors) for $15 \mathrm{~min}$. We used only PES rats that had undergone vaginal cellular morphology examination under a light microscope after daily vaginal lavage and exhibited at least three consecutive normal estrous cycles. These procedures are sammarized in Fig. 1.

The experiments were approved by the Animal Care and Use Committee of the Faculty of Agriculture, The University of Tokyo.

\section{Data analyses}

We performed power spectral analysis of heart rate variability (HRV) according to an established method 
described previously $[8-10,14,19]$. Basically, off-line analysis was performed on an ECG processor analyzing system (SRV-2W; Softron) using the recorded baseline and test ECG data stored on a computer. First, R waves were identified, and both heart rate (HR) and the R-R interval tachogram as the raw HRV were calculated. From the tachogram, data sets of 512 points were resampled at $80 \mathrm{~ms}$. We then applied each data set to a Hamming window and performed an FFT analysis to obtain the power spectrum of the fluctuation. We set the lowfrequency (LF) range of the power spectrum at 0.04-1.00 $\mathrm{Hz}$ and the high-frequency (HF) range at $1.00-3.00 \mathrm{~Hz}$. The LF region is considered to be a measure of both sympathetic and parasympathetic activity. The HF region is associated with respiratory sinus arrhythmia and is almost exclusively associated with parasympathetic activity. The LF/HF ratio was used as a measure of sympathovagal balance. The relative values of the LF and HF power compared with the respective baseline data were calculated to adjust baseline differences among individual subjects.

The videotape recording of each subject was replayed, and the duration of walking and rearing was summed as the total locomotor activity. Walking was defined as movements involving all four limbs. Rearing was defined as raising the front paws from the chamber floor and either placing them on the side of the chamber or in front of the body.

\section{Statistical analysis}

Data are displayed as means \pm standard error. The effects of female presentations on HR, spectral parameters of HRV (LF power, HF power, and LF/HF ratio), and total locomotor activity were analyzed in individually and socially housed male subjects separately using repeated measures one-way ANOVA with presentation as a within-subjects factor (i.e., WMB vs. OVX vs. PES). This was followed by a post hoc Dunnett's test in which the presentation of WMB was used as a control. The criterion for statistical significance was $P<0.05$ for all comparisons.

\section{Results}

In individually housed male rats, there were significant main effects of female presentations on LF power $\left(F_{2,14}=4.87, P<0.05\right)$ and HF power $\left(F_{2,14}=9.77, P<0.01\right)$. Post hoc tests showed that LF power and HF power in-
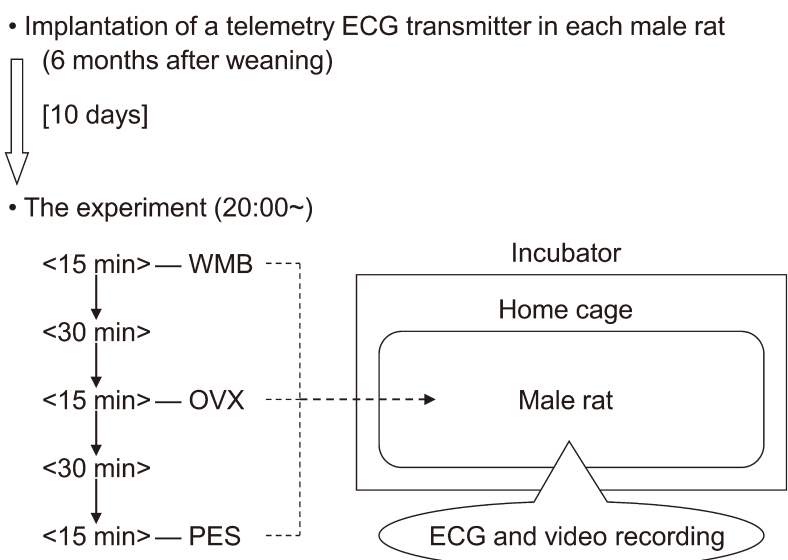

Fig. 1. A schematic diagram of our experimental schedule. A telemetry electrocardiogram (ECG) transmitter was implanted in each male rat 6 month after weaning. At least 10 days after surgery, the home cage of each subject was placed in an incubator, and each subject received a 15 -min presentation of a wire mesh box (WMB), an ovariectomized female rat in a wire mesh box (OVX), and a proestrous female rat in a wire mesh box (PES), with intervals of $30 \mathrm{~min}$. During each session of stimulus presentation $(15 \mathrm{~min})$, we performed ECG and video recording of subject male rats.

creased significantly in the presence of PES compared with WMB (LF power, $P<0.05$; HF power, $P<0.01$ ) (Figs. $2 \mathrm{~B}$ and $2 \mathrm{C}$ ). In contrast, there was no difference between OVX and WMB. In addition, the HR and LF/ HF ratios were almost identical throughout the experiment (Figs. 2A and 2D).

In socially housed male rats, there were significant main effects of female presentations on $\operatorname{HR}\left(F_{2,14}=86.2\right.$, $P<0.01)$, LF power $\left(F_{2,14}=114, P<0.01\right)$, HF power $\left(F_{2,14}=58.6, P<0.01\right)$, and LF/HF ratio $\left(F_{2,14}=32.1\right.$, $P<0.01)$. Post hoc tests showed that HR increased significantly $(P<0.01)$ (Fig. $2 \mathrm{~A})$ and that LF power, HF power, and $\mathrm{LF} / \mathrm{HF}$ ratio decreased significantly (LF power, $P<0.01$; HF power, $P<0.01$; LF/HF ratio, $P<0.01$ ) (Fig. 2B-D) in the presence of PES compared with WMB. In contrast, there was no difference between OVX and WMB (Fig. 3).

In both individually and socially housed male rats, the difference in total locomotor activity throughout the experiment showed no significance (individually, $F_{2,14}=0.79, P=0.48$; socially, $F_{2,14}=1.77, P=0.21$ ) (Table $1)$. 

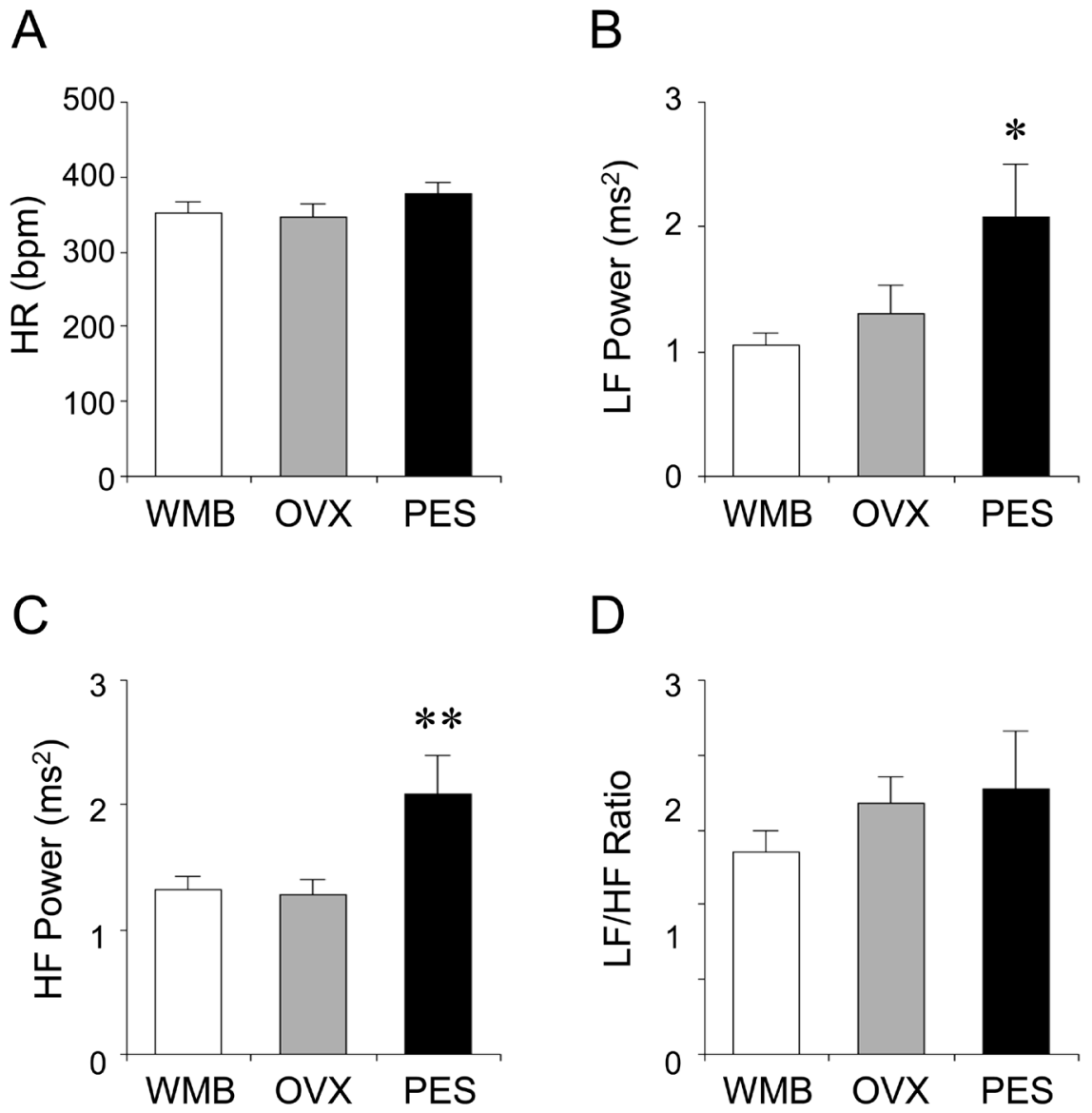

Fig. 2. Changes in heart rate and heart rate variability parameters in male rats housed individually after weaning $(n=8)$ induced by presentation of the empty wire mesh box (WMB), the ovariectomized female rat in the wire mesh box (OVX), and the proestrous female rat in the wire mesh box (PES). A, heart rate (HR); B, low-frequency (LF) power; $\mathrm{C}$, high-frequency $(\mathrm{HF})$ power; $\mathrm{D}, \mathrm{LF} / \mathrm{HF}$ ratio. Each bar represents the mean \pm standard error. ${ }^{*} * P<0.01$ vs. WMB. ${ }^{*} P<0.05$ vs. WMB.

\section{Discussion}

In this study, we investigated differences in HR, spectral parameters of HRV (LF power, HF power, and LF/ HF ratio), and total locomotor activity in adult male rats under two different post-weaning conditions when they encountered a sexually receptive female rat in a wire mesh box. Their HR and spectral parameters of HRV were altered markedly compared with those observed after presentation of the control. Moreover, the changes in these parameters differed according to post-weaning conditions, although total locomotor activity was almost constant during the experiments in rats under both postweaning conditions. These results show that autonomic nervous activity responses to the sexually receptive female rat in the wire mesh box were clearly distinguish- able between male rats housed individually and socially after weaning.

The results of this study suggest that the sexually receptive female rat could evoke distinct affective states in male rats depending on the males' housing conditions after weaning. Previous studies showed that an implanted telemetry system to record ECG of freely behaving animals and subsequent power spectral analysis of HRV are powerful tools for obtaining valid cardiovascular parameters of autonomic control of the heart in rats [8-10, 14, 19]. Analyses of HR and HRV in this study revealed that regardless of post-weaning conditions in male subjects, there was almost no difference in the autonomic nervous activity when the control (the wire mesh box) or the sexually nonreceptive female rat was presented to the males. In contrast, the sexually receptive 

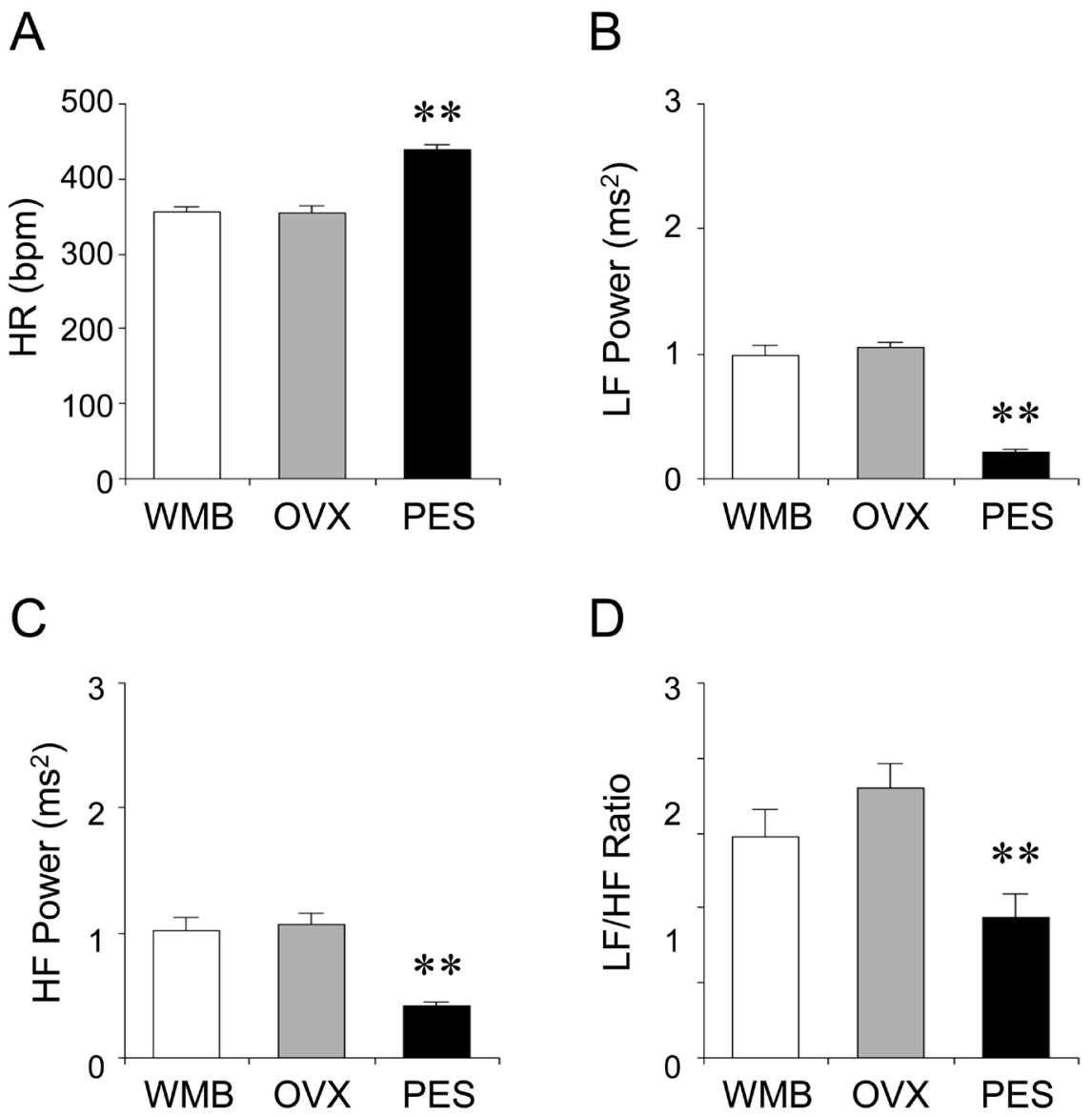

Fig. 3. Changes in heart rate and heart rate variability parameters in male rats housed socially after weaning $(n=8)$ induced by presentation of the empty wire mesh box (WMB), the ovariectomized female rat in the wire mesh box (OVX), and the proestrous female rat in the wire mesh box (PES). A, heart rate (HR); B, low-frequency (LF) power; $\mathrm{C}$, highfrequency (HF) power; $\mathrm{D}, \mathrm{LF} / \mathrm{HF}$ ratio. Each bar represents the mean \pm standard error. $* * P<0.01$ vs. WMB.

Table 1. Data of locomotor activity

\begin{tabular}{lcc}
\hline \multirow{2}{*}{ Presentation } & \multicolumn{2}{c}{ Locomotor activity (s) } \\
\cline { 2 - 3 } & Individually housed subjects & Socially housed subjects \\
\hline Wire mesh box & $43.4 \pm 5.06$ & $35.3 \pm 4.52$ \\
Ovariectomized female & $36.1 \pm 7.99$ & $31.8 \pm 4.16$ \\
Proestrous female & $36.7 \pm 7.35$ & $30.7 \pm 3.66$ \\
\hline
\end{tabular}

Values are means \pm standard error. Eight male rats were used in each group.

female rat caused the following distinct shifts in HR and spectral parameters of HRV in male subjects according to their post-weaning housing conditions compared with the control presentation. In post-weaning individually housed male rats, both the LF power and HF power increased significantly with no change in the HR or LF/ HF ratio, indicating that both sympathetic and parasympathetic activity increased with no change in sympa- thovagal balance. In post-weaning socially housed male rats, a significant increase in the HR and significant decreases in the LF power, HF power, and LF/HF ratio were observed, indicating that both sympathetic and parasympathetic activity decreased with predominance of parasympathetic activity. These two patterns of shifts closely resemble results obtained from our previous study of changes in the autonomic nervous activity with 
regard to classical appetitive conditioning in male rats. Specifically, that autonomic changes associated with the reward-expecting state evoked by the conditioned stimulus (a contextual cage-switch stimulus) correspond to the above-mentioned changes observed in male rats housed individually after weaning, and that autonomic changes associated with the reward-receiving state evoked by the unconditioned stimulus (e.g., delivery of food or sucrose solution after deprivation) correspond to the above-mentioned changes observed in male rats housed socially after weaning [9]. In addition, although HRV can be influenced by physical activity [2], it seems to be unnecessary to take this effect into consideration in this study because there was no difference in total locomotor activity between the subject groups. Therefore, it is probable that male subjects housed individually after weaning might recognize the presented sexually receptive female rat as a relatively uncertain reward and respond as if receiving a neutral conditioned stimulus, while male subjects housed socially after weaning might recognize the same female rat as a real reward and respond as if receiving food or sucrose solution after deprivation. Namely, it is plausible that male subjects housed socially after weaning might recognize the presented sexually receptive female rat as reward more directly and substantially than male subjects housed individually after weaning. Accordingly, post-weaning individual housing might reduce the ability of male rats to recognize a sexually receptive female rat as a reward.

These data suggest that post-weaning individual housing substantially influences affective states in male rats during sexual encounters. One explanation for this phenomenon is that post-weaning individual housing might cause alteration in the recognition of social cues from sexually receptive female rats. Increased aggression is one of the most well-known abnormal male-male social interactions observed in adult male rats housed individually after weaning [5]. An earlier study demonstrated that post-weaning individual housing might not only increase the level of aggressiveness, but also might result in abnormal attack patterns and deficits in social communication in adult male rats [17]. Another recent study showed that these abnormal forms of aggression may be developed on a background of increased behavioral, endocrine, and autonomic arousal during aggression, all of which may be characterized by a mismatch between provocation and response, indifference toward species-specific rules, and/or insensitivity toward social signals of the opponent [18]. Therefore, it is plausible that post-weaning individual housing diminishes the ability of male rats to appropriately recognize probable social cues from female rats; namely, olfactory, auditory, and visual signals. However, further studies on the basis of examination of not only male-female but also male-male relationships are necessary to clarify this issue.

In conclusion, our data demonstrate that the postweaning housing condition can dramatically influence autonomic responses in adult male rats to sexually receptive female rats, suggesting that different affective states are evoked during sexual encounters depending on malemale social interactions after weaning. The remarkable alteration caused by post-weaning individual housing may be ascribed to a reduced ability of male rats to recognize sexually receptive female rats as a reward, suggesting alteration of the reward system during sexual encounters induced by deficiency in intermale social communication after weaning. Post-weaning individual housing has been demonstrated to evoke alteration of sexual behaviors in male rats $[1,3,6]$. The results of this study may add new physiology-based evidence of developmental functional alteration in male rats with regard to male-female relationships induced by deficiency of male-male social interaction after weaning.

\section{References}

1. Bakker, J., Vanophemert, J., and Slob, A.K. 1995. Postweaning housing conditions and partner preference and sexual behavior of neonatally ATD-treated male rats. Psychoneuroendocrinology 20: 299-310. [Medline] [CrossRef]

2. Bernardi, L., Valle, F., Coco, M., Calciati, A., and Sleight, P. 1996. Physical activity influences heart rate variability and very-low-frequency components in Holter electrocardiograms. Cardiovasc. Res. 32: 234-237. [Medline] [CrossRef]

3. Brotto, L.A., Gorzalka, B.B., and Hanson, L.A. 1998. Effects of housing conditions and $5-\mathrm{HT}_{2 \mathrm{~A}}$ activation on male rat sexual behavior. Physiol. Behav. 63: 475-479. [Medline] [CrossRef]

4. Burgdorf, J., Kroes, R.A., Moskal, J.R., Pfaus, J.G., Brudzynski, S.M., and Panksepp, J. 2008. Ultrasonic vocalizations of rats (Rattus norvegicus) during mating, play, and aggression: behavioral concomitants, relationship to reward, and self-administration of playback. J. Comp. Psychol. 122: 357-367. [Medline] [CrossRef]

5. Day, H.D., Seay, B.M., Hale, P., and Hendricks, D. 1982. Early social deprivation and the ontogeny of unrestricted social-behavior in the laboratory rat. Dev. Psychobiol. 15: 47-59. [Medline] [CrossRef]

6. Dunlap, J.L., Zadina, J.E., and Gougis, G. 1978. Prenatal 
stress interacts with prepuberal social isolation to reduce male copulatory behavior. Physiol. Behav. 21: 873-875. [Medline] [CrossRef]

7. Fone, K.C.F. and Porkess, M.V. 2008. Behavioural and neurochemical effects of post-weaning social isolation in rodents - Relevance to developmental neuropsychiatric disorders. Neurosci. Biobehav. Rev. 32: 1087-1102. [Medline] [CrossRef]

8. Hashimoto, M., Kuwahara, M., Tsubone, H., and Sugano, S. 1999. Diurnal variation of autonomic nervous activity in the rat: investigation by power spectral analysis of heart rate variability. J. Electrocardiol. 32: 167-171. [Medline] [CrossRef]

9. Inagaki, H., Kuwahara, M., and Tsubone, H. 2005. Changes in autonomic control of heart associated with classical appetitive conditioning in rats. Exp. Anim. 54: 61-69. [Medline] [CrossRef]

10. Inagaki, H., Kuwahara, M., and Tsubone, H. 2004. Effects of psychological stress on autonomic control of heart in rats. Exp. Anim. 53: 373-378. [Medline] [CrossRef]

11. Inagaki, H., Kuwahara, M., Tsubone, H., and Mori, Y. 2013. The effect of post-weaning individual housing on $50-\mathrm{kHz}$ calls emitted from male rats to sexually receptive female rats. Physiol. Behav. 110-111: 30-33. [Medline] [CrossRef]

12. Knutson, B., Burgdorf, J., and Panksepp, J. 2002. Ultrasonic vocalizations as indices of affective states in rats. Psychol. Bull. 128: 961-977. [Medline] [CrossRef]

13. Kreibig, S.D. 2010. Autonomic nervous system activity in emotion: A review. Biol. Psychol. 84: 394-421. [Medline] [CrossRef]

14. Kuwahara, M., Yayou, K., Ishii, K.J., Hashimoto, S., Tsub- one, H., and Sugano, S. 1994. Power spectral analysis of heart rate variability as a new method for assessing autonomic activity in the rat. J Electrocardiol 27: 333-337. [Medline] [CrossRef]

15. Luciano, D. and Lore, R. 1975. Aggression and social experience in domesticated rats. J. Comp. Physiol. Psychol. 88: 917-923. [Medline] [CrossRef]

16. McGinnis, M.Y. and Vakulenko, M. 2003. Characterization of $50-\mathrm{kHz}$ ultrasonic vocalizations in male and female rats. Physiol. Behav. 80: 81-88. [Medline] [CrossRef]

17. Toth, M., Halasz, J., Mikics, E., Barsy, B., and Haller, J. 2008. Early social deprivation induces disturdbed social communication and violent aggression in adulthood. Behav. Neurosci. 122: 849-854. [Medline] [CrossRef]

18. Toth, M., Mikics, E., Tulogdi, A., Aliczki, M., and Haller, J. 2011. Post-weaning social isolation induces abnormal forms of aggression in conjunction with increased glucocorticoid and autonomic stress responses. Horm. Behav. 60: 28-36. [Medline] [CrossRef]

19. Towa, S., Kuwahara, M., and Tsubone, H. 2004. Characteristics of autonomic nervous function in Zucker-fatty rats: investigation by power spectral analysis of heart rate variability. Exp. Anim. 53: 137-144. [Medline] [CrossRef]

20. Veenema, A.H. 2009. Early life stress, the development of aggression and neuroendocrine and neurobiological correlates: What can we learn from animal models? Front. Neuroendocrinol. 30: 497-518. [Medline] [CrossRef]

21. Wongwitdecha, N. and Marsden, C.A. 1996. Social isolation increases aggressive behaviour and alters the effects of diazepam in the rat social interaction test. Behav. Brain. Res. 75: 27-32. [Medline] [CrossRef] 